\title{
Near Critical Gas Condensate Relative Permeability of Carbonates
}

\author{
Huseyin Calisgan $^{1}$ and Serhat Akin ${ }^{*}, 2$ \\ ${ }^{1}$ TPAO Research Center, Ankara, Turkey \\ ${ }^{2}$ Petroleum and Natural Gas Engineering Department, Middle East Technical University, Ankara, Turkey
}

\begin{abstract}
Typical gas condensate fields contain a gas/liquid system during depletion. Such systems are difficult to model experimentally because they exhibit near-miscible behavior at high pressure and temperature. One way to simplify laboratory experimentation is to use a binary retrograde condensate fluid and to adjust temperature to control miscibility. A series of relative permeability test were conducted on a moderate-permeability carbonate core using methanol/n-hexane at near miscible conditions in the presence of immobile water. Potassium carbonate was added to the water to prevent miscibility with methanol. The experiments used a pseudo-steady-state technique under conditions similar to the near well region of a carbonate gas-condensate reservoir. The flow of gas and condensate at different force ratios was investigated. Relative permeabilities were obtained by matching historical production and pressure data using a coreflood simulator. It was observed that relative permeability depended on fluid composition and flow rate as well as initial condensate and water saturations. As the wetting phase (condensate) flow rate increased or interfacial tension decreased, relative permeability versus wetting phase saturation curves shifted towards lower wetting phase saturations. It was found that a simple three-parameter mathematical model that depends on a new dimensionless number called condensate number successfully modeled the gas-condensate relative permeability data. The developed model resulted in a good agreement with published gas-condensate relative permeability data as well as end point relative permeabilities and saturations. The relative permeability behavior as a function of IFT highly resembles the one observed in sandstones.
\end{abstract}

Keywords: Condensate, relative permeability, model, condensate number.

\section{INTRODUCTION}

In a gas condensate reservoir, there are many important differences between the flow regimes in the regions close to and far from the well. These different flow regimes are reflected in the requirements for relative permeability data for the reservoir and near well regions. Far from the well, flow rates are low, and liquid mobility is usually less important, except in reservoirs containing very rich light component fluids. In the near well region, both liquid and gas phases are mobile, flow rates are high, and the liquid mobility is important. At initial reservoir conditions the hydrocarbon fluids are mostly present at near-critical conditions. Consequently, the physical properties of the oil phase are very similar, and the interfacial tension between oil and gas is very low. During the production phase of gas condensate reservoir multi phase fluid problem becomes important below dew point pressure. One of the important multi-phase fluid flow problems at near critical conditions is condensate drop out in the vicinity of wells in gas condensate reservoirs. This drop out causes an apparent skin resistance at the well bore that impairs the production capacity of well.

The effect of near-criticality on the relative permeability is still an unsolved issue in reservoir engineering. Experimental studies published in the literature indicate a trend from immiscible to miscible relative permeability curves as the interfacial tension approaches zero. Unfortunately, there

*Address correspondence to this author at the Petroleum and Natural Gas Engineering Department, Middle East Technical University, Ankara, Turkey; E-mail: serhat@metu.edu.tr is no consensus on how near miscibility changes relative permeability curves and which parameters control this change. Some investigators [1-3] have found that the relative permeability to the non-wetting phase is affected more easily, whereas others observed a greater increase of the relative permeability to the wetting phase compared with the relative permeability to the non-wetting phase $[4,5]$. More importantly, some studies did not report an effect of interfacial tension at all $[6,7]$. Equally contradicting are the reports on the effect of flow velocity on near-miscible relative permeability. Some investigators find no effect $[8,9]$, whereas others do [2, 10]. In addition, Henderson et al. [3] have reported that relative permeability is only affected by the flow velocity if the fluids enter the porous medium as a single, homogeneous phase, and subsequently, are allowed to separate into two phases inside the pores.

There are two conflicting views on which mechanism controls the change in relative permeability. Many authors argue that a low interfacial tension affects relative permeability through the ratio between viscous forces and capillary forces, as denoted by the capillary number [1, 2, 10-14]. Most of these authors, however, suggest that there is a threshold interfacial tension below which the capillarynumber dependence becomes important $[1-3,11,12]$. Relative permeability data is usually interpreted in terms of the interfacial tension alone $[4,5,15-18]$. In two cases, this was done in view of the fact that a transition from partial wetting to complete wetting, as predicted by Cahn [19], may affect the mobility of both phases $[5,9]$. The influence of such a transition cannot be described in terms of the capillary num- 
ber, because it is directly induced by a change in the interfacial tension between the near-miscible phases.

According to current understanding of the flow behavior in gas condensate systems, at least two flow regimes may be considered: one corresponding to conditions away from the critical point, where IFT's are relatively high, and another to conditions near the critical point, where IFT's are very low [20]. Far from the critical point, the relative permeability curves show considerable curvature and appreciable residual saturations. Near the critical point, the IFT reaches very low values and the relative permeability curves become progressively straighter, with the residual saturations diminishing. In the limit of zero IFT, the curves become straight lines, the residual saturations vanish, and the sum of the relative permeabilities is unity for all saturations. This scenario is supported by experimental studies [11,21].

The relative permeability data of the gas and condensate can be modeled by an empirical formula representing the interfacial tension [22], but, it has been known that the relative permeability data in general actually depend on the ratio of forces on the trapped phase, which can be expressed as either a capillary number or bond number [23]. This has been recognized in recent years to be true for gas-condensate relative permeability data $[3,10]$. A common approach is to interpolate between the limit at capillary-dominated flow, in which the capillary number is equal to zero and the limit that is approached at an infinitely high capillary number, corresponding to viscous-dominated flow representing the immiscible and miscible limits respectively. A critical review of interpolation based mathematical relative permeability models is presented by Blom and Hagoort [13]. They concluded that an appropriate weighting function should enable adjustment of both steepness of change and the capillary number at which the relative permeability curve is halfway between the limiting curves. A simple two-parameter capillary trapping model that is a generalization of the approach by Delshad et al. [24] was proposed by Pope et al. [25]. In this model, a general scheme for computing the gas and condensate relative permeability data as a function of the trapping number was generated.

As can be seen from the aforementioned discussion the condensate relative permeability still is a topic of discussion. Most of the literature concentrates around sand packs and sandstones. Therefore, the objective of this work is to provide conclusive experimental evidence of the effect of interfacial tension, flow velocity and presence of immobile water saturation on near-miscible relative permeability of carbonates. To achieve this goal, unsteady state displacement experiments were conducted using a carbonate core plug. Methanol-hexane binary liquid system that exhibits a critical point at atmospheric pressure and at a temperature of $33.5^{\circ} \mathrm{C}$ and a methanol fraction of 0.56 was used. The interfacial tension between the fluids was controlled through adjustment of the temperature, whereas, the flow velocity was regulated by adjusting the injection rate. The effect of immobile water saturation is also studied by employing a novel laboratory technique involving addition of $\mathrm{K}_{2} \mathrm{CO}_{3}$ to watermethanol mixture. The results are interpreted with a three parameter mathematical model that depends on a new dimensionless number called condensate number. It was concluded that the developed model successfully models the gas-condensate relative permeability data as a good agreement with published gas-condensate relative permeability data as well as end point relative permeabilities and saturations were observed.

\section{MATHEMATICAL MODEL}

The fundamental problem with condensate buildup in the reservoir is that capillary forces can keep the condensate in the pores if the forces displacing the condensate do not exceed the capillary forces. The pressure forces in the displacing gas phase and the buoyancy force on the condensate exceed the capillary force on the condensate, the condensate saturation will be reduced and the gas relative permeability increased. Brownell and Katz [23] and others recognized early on that the residual oil saturation should be a function of the ratio of viscous to interfacial forces and defined a capillary number to capture this ratio.

$$
N_{C}=\frac{\vec{u} \mu_{d}}{\sigma_{d u}}
$$

In some cases, buoyancy forces can contribute significantly to the total force on the trapped phase. To quantify this effect, the bond number was introduced and it also takes different forms in the literature.

$N_{B}=\frac{\vec{k} g\left(\rho_{d}-\rho_{u}\right)}{\sigma_{d u}}$

For special cases such as vertical flow, the force vectors are collinear and one can just add the scalar values of the viscous and buoyancy forces and correlate the residual oil saturation with this sum, or in some cases one force is negligible compared to the other force and just the capillary number or Bond number can be used by itself. This is the case with most laboratory studies including the recent ones [3, 10]. However, in general the forces on the trapped phase are not collinear in reservoir flow and the vector sum must be used. A generalization of the capillary and Bond numbers was derived by Jin [26] and called the trapping number. The trapping number for phase displaced by phase is defined as follows: The trapping number [25], $\mathrm{N}_{\mathrm{t}}$, uses generalized form of the capillary, $\mathrm{N}_{\mathrm{c}}$ and bond numbers, $\mathrm{N}_{\mathrm{b}}$ as

$$
N_{T}=\frac{\overrightarrow{\vec{k}}\left(\vec{\nabla} \Phi_{d}+g\left(\rho_{d}-\rho_{u}\right)+\vec{\nabla} D\right)}{\sigma_{d u}}
$$

Similarly a dimensionless number, $\mathrm{N}_{\mathrm{k}}$, given by the sum of capillary, $\mathrm{N}_{\mathrm{c}}$, and bond numbers, $\mathrm{N}_{\mathrm{b}}$ can be obtained. Note that this dimensionless number (condensate number) is more sensitive to temperature changes compared to trapping number since $\mathrm{N}_{\mathrm{k}}$ reflects both viscosity and density changes. Moreover, it minimizes the measurement errors resulting from the use of inaccurate pressure transducers or gauges.

$$
N_{K}=\frac{\vec{u} \mu_{d}+\overrightarrow{\vec{k}} g\left(\rho_{d}-\rho_{u}\right)}{\sigma_{d u}}
$$

The residual condensate saturation is defined following Pope et al. [25], but rather than using two parameters a three parameter approach is preferred. The additional parameter adds improved matching abilities. 
$S_{d r}=\min \left(S_{d}, S_{d r}^{h i g h}+\frac{S_{d r}^{l o w}-S_{d r}^{h i g h}}{c+a\left(N_{K}\right)^{b}}\right)$

Here superscripts high and low refer to residual saturations of the gas and condensate. High value of $\mathrm{S}_{\mathrm{dr}}$ is typically zero. The end point relative permeabilities of each phase, which increases as the condensate number increases and can be correlated using the following equation:

The next step is to correlate the endpoint relative permeability of each phase, which increases in a very predictable way as the condensate number increases and can be correlated using the following equation:

$k_{r d}^{0}=k_{r d}^{0 l o w}+\frac{S_{d r}^{l o w}-S_{d r}}{S_{d r}^{l o w}-S_{d r}^{\text {high }}}\left(k_{r d}^{0 \text { ohigh }}-k_{r d}^{\text {olow }}\right)$

One other approach to this problem is to assume a simple function such as a Corey-type relative function. This requires correlating the Corey exponent with trapping number. However, not all the relative permeability data can be fit with a Corey-type model, so a generalized approach is proposed by using Eqn. 7 that is modified after Pope [25]. Again the performance of the model is controlled by the parameters used in Eqn. 5.

$\log \left(k_{r d}\right)=\log k_{r d}^{0}+\log \left(\bar{S}_{d}\right)+\frac{\log \left(\frac{k_{r d}}{k_{r d}^{0}}\right)^{\text {low }}-\log \left(\bar{S}_{d}\right)}{c+a\left(N_{K}\right)^{b}}$

where $k_{r d}^{0}$ is end point relative permeability for a given trapping number and saturation, $k_{r d}^{\text {low }}$ and $k_{r d}^{\text {olow }}$ are relative permeability and end point relative permeability at low trapping number. Saturations are normalized as:
$\bar{S}_{d}=\frac{S_{d}-S_{d r}}{1-\sum_{d=1}^{n} S_{d r}}$

where " $n$ " is number of the phases present, $\mathrm{S}_{\mathrm{d}}$ is saturation and $\mathrm{S}_{\mathrm{dr}}$ is residual saturation.

We show the comparisons (Fig. 1) with end point relative permeability data and experimental non-wetting phase data for various porous media using data from Hartman and Cullick [17] and Henderson et al. [3]. Hartman and Cullick [17] obtained as methane and butane; $\left(\mathrm{C}_{1} / \mathrm{n}-\mathrm{C}_{4}\right)$ relative permeability data from steady state slim tube sand pack experiments. On the other hand Henderson et al.'s [3] data was obtained using Berea sandstone and methane and butane fluid pair. It was observed that the mathematical model correlates well with literature data. The constant " $a$ " is quite different due to use of various types of porous media of differing wettability; however, the general trend of increasing endpoint relative permeability with increasing condensate number is consistent.

As pointed out above, the best starting point for understanding and modeling relative permeability data as a function of interfacial tension is the relationship between the residual saturations and condensate number (or its special cases of capillary number or Bond number when appropriate to the experimental conditions). For this reason, the comparison of the model with experimental data of normalized residual saturation versus condensate number is shown in Fig. (2). Bardon and Langeron [11] used sandstone and $C_{7}$-rich liquid for measurement of relative permeability curves by unsteady state injection method at $S_{\text {wirr }}=0.35$. Delshad [24]; however, run the tests at $\mathrm{S}_{\text {wirr }}=0.40$. Henderson et al. [3] used methane and n-butane in steady state displacement tests

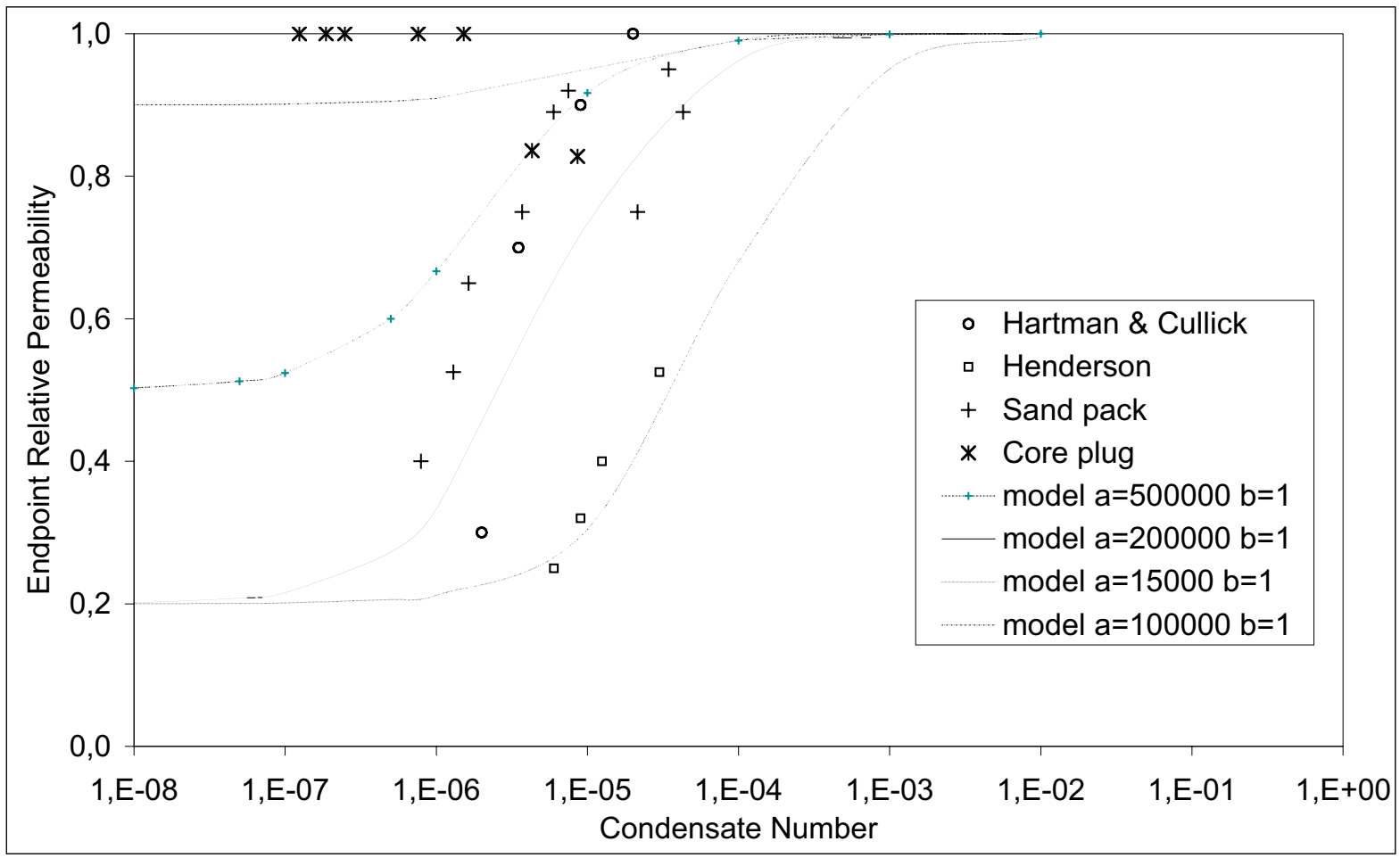

Fig. (1). Comparison of model and literature end point relative permeability data. 


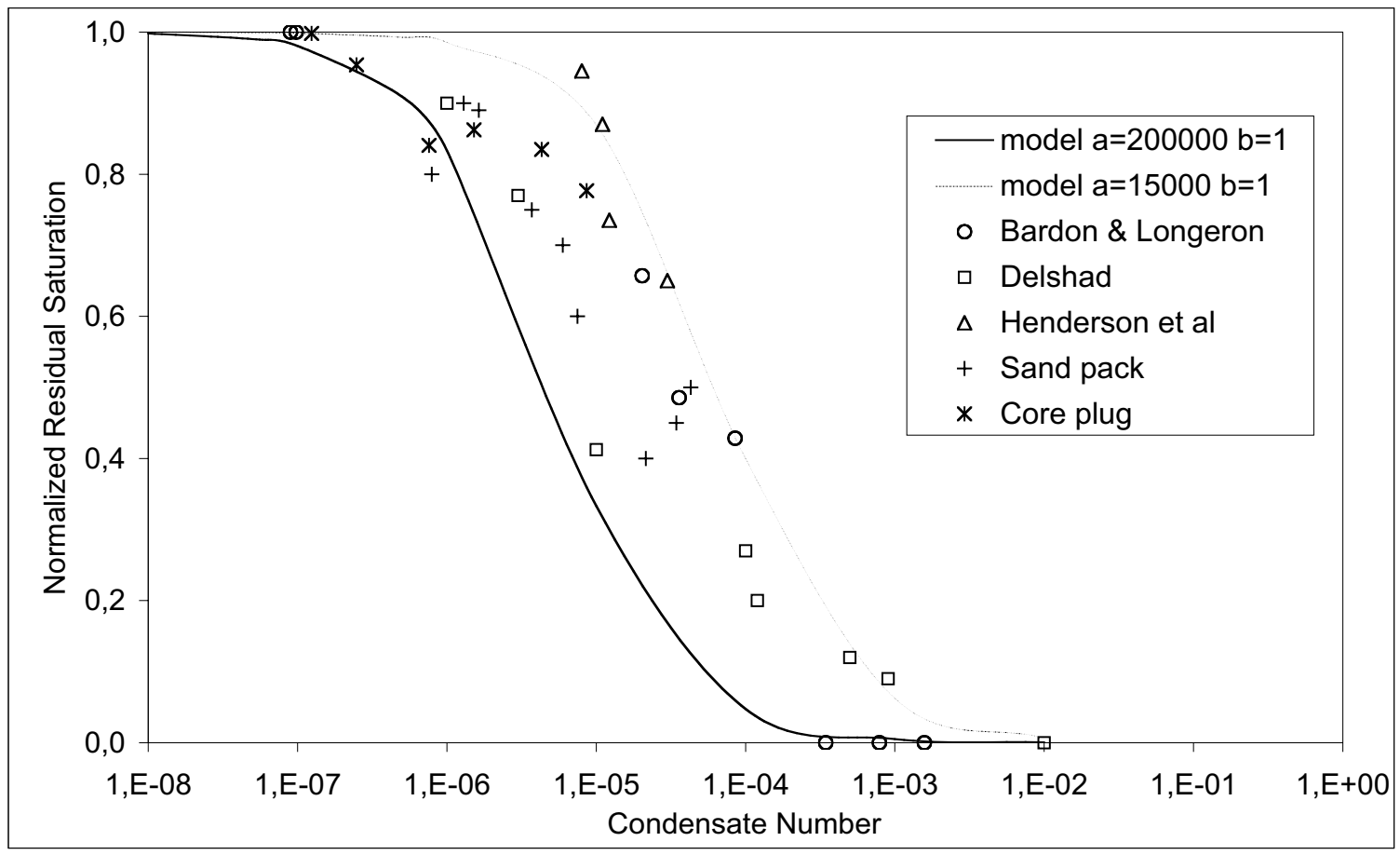

Fig. (2). Comparison of model and literature residual saturation data.

at the non-wetting phase residual saturation, $\mathrm{S}_{\mathrm{nwr}}=0.29$. In order to compare all data saturations are normalized between 0 and 1 for residual saturations. The constant which can be interpreted as a porous medium property again changes due to high wettability differences and the constant " $b$ " is around 1. As can be seen the proposed model matches well with the literature test results. The conclusion is that one must measure the residual saturations for the wetting state and rock of interest to get useful results that can be accurately applied to a particular reservoir state. In particular, if there are three phases in the reservoir such as there are with gas condensates then, to ensure the correct wetting and spreading state in the rock, three phases need to be in the laboratory core even if one of the phases such as the brine is always at residual saturation.

The new model results for gas relative permeability versus gas saturation are given in Fig. (3) for different condensate numbers. As the condensate number become high (between $10^{-2}$ and $10^{-3}$ ), gas relative permeability gas saturation approaches a linear relationship. For low condensate number, $\mathrm{N}_{\mathrm{k}}$ gas relative permeability, $\mathrm{k}_{\mathrm{rg}}$ versus gas, $\mathrm{S}_{\mathrm{g}}$ is not linear.

\section{EXPERIMENTAL}

\section{Fluids}

The gas condensate fields concern a gas/liquid system during depletion stage. Such systems have a characteristics behavior of near-miscible at high pressure and temperatures, which complicates laboratory experiments. In this regard, binary liquid/liquid [14] systems are more suitable candidates compared to gas/liquid [27] ones in a laboratory displacement and phase behavior test. The phase behavior of a liquid/liquid system is hardly susceptible to changes in pressure. The interfacial tension of liquid/liquid systems varies much more strongly than that of gas/liquid systems, so that a smaller temperature range enables assessment of the influence of interfacial tension. Yet another advantage of using this synthetic gas condensate fluid pair is easy to handle, better characterization, and no need to work at very high temperatures and pressures.

Near-miscible fluids are special in a thermodynamic sense because they are found at conditions that are close to a critical point of the fluid system. The interfacial tension between near-miscible phases is low. Near-miscibility is found in two types of fluids systems as gas/liquid system i.e. gaseous and a liquid phase or as liquid/liquid system i.e. two liquid phases at specific pressure, temperature and compositions. The fluid system should satisfy two conditions: the fluid system should be near-miscible under the conditions close to room temperature and atmospheric pressure and the behavior of its physical properties as a function of the distance to the critical point should be analogous to that of a gas/condensate fluid. Methanol/n-hexane binary liquid mixture was used throughout the test runs. In such a system, the methanol-rich phase is the wetting fluid. Methanol is the denser, heavier and more viscous phase, thus playing the role of the liquid hydrocarbon phase in a typical gas condensate reservoir. The hexane rich phase in equilibrium with the methanol rich phase represents the non wetting phase. The main advantage of using such a system is that experiments can be performed at less extreme conditions, as the methanol/hexane system shows a critical point at atmospheric pressure and at a temperature of $33.5^{\circ} \mathrm{C}$. Another advantage is that the phase behavior of the binary liquid is not susceptible to the pressure changes. Therefore, methanol-rich phase acts as a liquid (condensate) and the hexane-rich phase plays the role of gaseous phase in the gas/condensate fluid. The literature data on the critical points in the last 50 years are contradictory and indicates the following ranges [14]: 


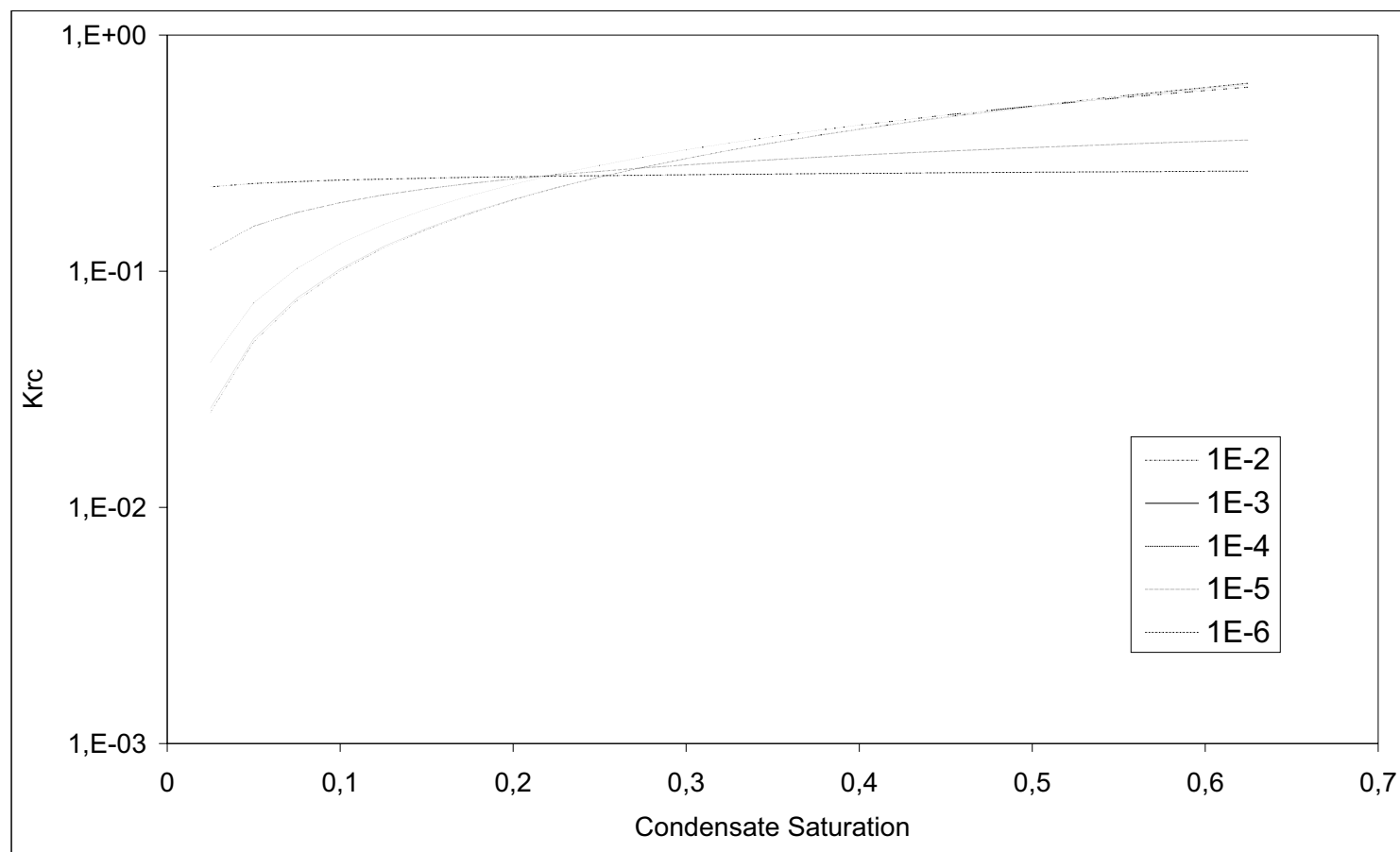

Fig. (3). Gas condensate relative permeability at different condensate numbers.

$33.2^{\circ} \mathrm{C}<\mathrm{Tc}<36.5^{\circ} \mathrm{C}$

$0.55<$ Methanol $\mathrm{x}_{\mathrm{c}}<0.57$

A disadvantage of the methanol/hexane system is that its phase behavior is very sensitive to minor amounts of impurities, particularly water that requires extra precautions in the handling of the fluids, to ensure the purity of the mixture. The methanol that was used in the experiments was extra dried (maximum, $0.01 \%$ water), with an overall purity that was better than $99.5 \%$. The purity of the n-hexane was better than $99.0 \%$ with less then $0.02 \%$ water. The purity of $n-$ hexane and methanol enabled a critical temperature of $33.5 \pm$ $0.01{ }^{\circ} \mathrm{C}$ and a critical methanol mole fraction of $0.56 \pm 0.02$ [28].

\section{Experimental Equipment and Procedure}

The experimental equipment consisted of constant displacement pumps, a constant temperature Hassler type core holder and a separator (Fig. 4). The production is measured using an automated fractional collector. The system is kept inside a constant temperature oven to ensure isothermal operation. The thermostatic air oven controlled the temperature within $0.01^{\circ} \mathrm{C}$. Thus during an experiment the properties of the fluids remain constant within $0.1 \%$ whereas the interfacial tension changes within $1.5 \%$. All tubing and tubing connections were made of steel to avoid chemical reactions with methanol and hexane. The pressure difference is measured as a function of time using a pressure differential transducer that is connected to a data logger. Prior to each test length, diameter and weight as well as porosity and absolute permeability of the core plug are measured (Table 1). Then the core is fully saturated with methanol rich phase so that any residual n-hexane would dissolve. The temperature of the thermostatic oven is set to desired temperature. Following that the methanol rich phase is allowed to come in to equilibrium with n-hexane rich phase. Finally, n-hexane rich mixture is injected into the core at the desired rate. Table 2 reports the conditions at each temperature (i.e. interfacial ten-

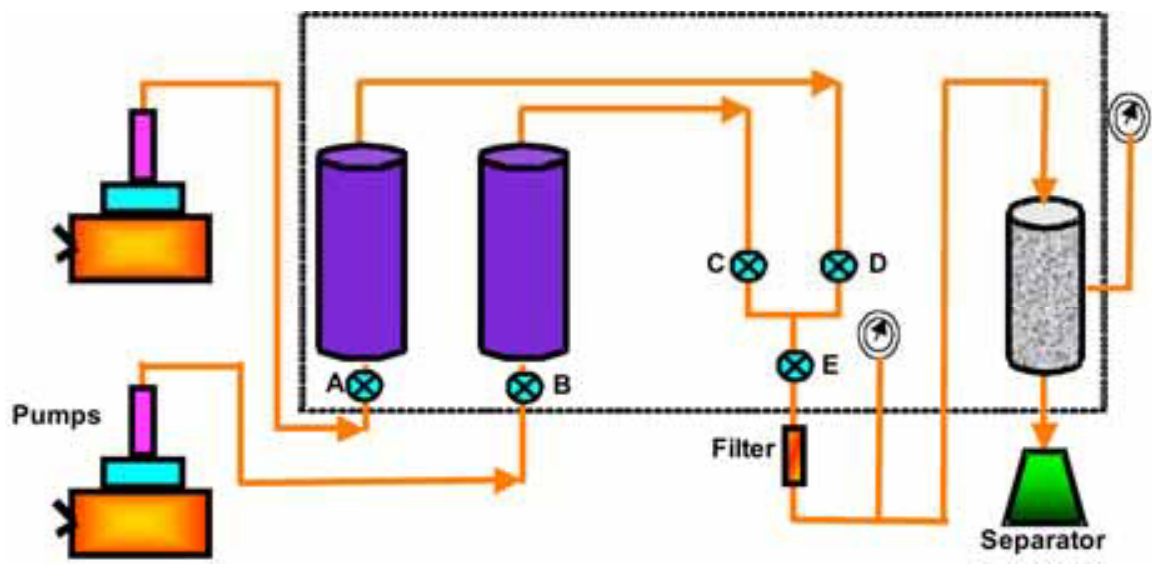

Fig. (4). Experimental setup. 
sion and flow rate changes). Once the pressure drop across the core plug and the production data as a function of time is measured the data can be interpreted using a coreflood simulator [29]. In between the experiments the core plug was placed in an oven and dried at $60{ }^{\circ} \mathrm{C}$ for two days to remove traces of methanol and n-hexane. For tests with initial water saturation, the core was first flooded with water that contained $5 \%$ by weight $\mathrm{K}_{2} \mathrm{CO}_{3}$ to ensure immiscibility of methanol in water. A few crystals of $\mathrm{K}_{2} \mathrm{Cr}_{2} \mathrm{O}_{7}, \mathrm{CuSO}_{4} \cdot 5 \mathrm{H}_{2} \mathrm{O}$ and a spatula tip of methyl red were added to the produced liquid to color the water and methanol differently so that phase separation could be visualized. The above procedure resulted in initial water saturations around $24.5 \%$ (Table 2 ).

Table 1. Testing Fluid and Core Plug Properties

\begin{tabular}{|l|l|}
\hline Test Fluid & Methanol - n-Hexane \\
\hline & $56 \% \quad-44 \%$ \\
\hline Methanol density, gm/cc & 0.8 \\
\hline Hexane density, gm/cc & 0.66 \\
\hline Well & N. Marmara - 1 \\
\hline Plug depth, m & 1155.1 \\
\hline Core length, cm & 6.82 \\
\hline Diameter, cm & 3.78 \\
\hline K air, md & 18.56 \\
\hline Grain density, gm/cc & 2.7 \\
\hline
\end{tabular}

Table 2. Experimental Conditions

\begin{tabular}{|c|c|c|c|c|c|c|c|}
\hline$\#$ & $\mathbf{T}\left({ }^{\circ} \mathbf{C}\right)$ & $\mathbf{I F T}(\mathbf{m} \mathbf{N} / \mathbf{m})$ & $\mathbf{Q}(\mathbf{c c} / \mathbf{h r})$ & $\mathbf{N}_{\mathbf{k}}$ & $\mathbf{S}_{\mathbf{w i}}$ & $\mathbf{S}_{\mathbf{n w}}$ & $\mathbf{S}_{\text {wet }}$ \\
\hline \hline 1 & 32.8 & 0.01 & 100 & $8.62 \mathrm{E}-6$ & 0 & 0.7617 & 0.2383 \\
\hline 2 & 32.8 & 0.01 & 75 & $6.46 \mathrm{E}-6$ & 0 & 0.7683 & 0.2317 \\
\hline 6 & 32.8 & 0.01 & 50 & $4.31 \mathrm{E}-6$ & 0 & 0.7946 & 0.2054 \\
\hline 3 & 30.1 & 0.059 & 100 & $1.52 \mathrm{E}-6$ & 0 & 0.8835 & 0.1165 \\
\hline 4 & 30.1 & 0.059 & 50 & $7.59 \mathrm{E}-7$ & 0 & 0.9016 & 0.09840 \\
\hline 5 & 18 & 0.3933 & 100 & $2.48 \mathrm{E}-7$ & 0 & 0.9189 & 0.08110 \\
\hline 7 & 18 & 0.3933 & 50 & $1.24 \mathrm{E}-7$ & 0 & 0.9245 & 0.0755 \\
\hline 8 & 18 & 0.3933 & 100 & $2.48 \mathrm{E}-7$ & 0.245 & 0.5922 & 0.1633 \\
\hline 9 & 18 & 0.3933 & 50 & $1.24 \mathrm{E}-7$ & 0.243 & 0.6175 & 0.1380 \\
\hline 10 & 32.8 & 0.01 & 100 & $8.62 \mathrm{E}-6$ & 0.242 & 0.5858 & 0.1697 \\
\hline 11 & 32.8 & 0.01 & 50 & $4.31 \mathrm{E}-6$ & 0.245 & 0.6004 & 0.1551 \\
\hline
\end{tabular}

\section{Core Properties}

A carbonate core plug drilled from a gas field (North Marmara, Turkey) was used in the relative permeability tests. The core plug was relatively homogeneous apart from occasional small vugs (Fig. 5) and it didn't contain any visible fissures or fractures. The same core plug was used in all experiments to minimize the effects of changing porous medium. The physical properties of the core plug are given in Table 1.
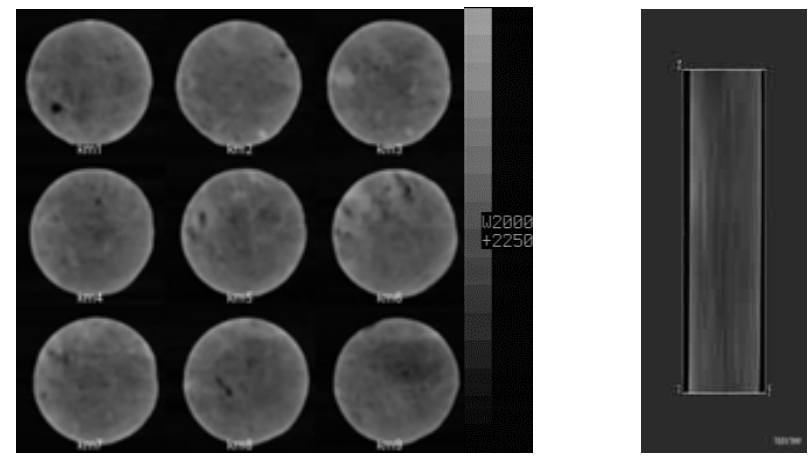

Fig. (5). CT slices (left) and vertical cross section of the reconstructed carbonate core plug used in the experiments.

\section{EXPERIMENTAL RESULTS}

Relative permeability measurements were carried out at 3 different temperatures and 3 different injection rates corresponding to 3 IFT's. Four of these experiments were repeated for immobile water saturation presence. The following sections describe the effects of flow rate and interfacial tension on the near critical condensate relative permeability data.

\section{Effect of Flow Rate}

When rate sensitivity was investigated, it was observed that the relative permeability of the gas phase (i.e. n-hexane) was particularly sensitive to velocity, and increased with increasing velocity at both values of IFT, as shown in Figs. $(6,7)$. As the velocity changed from $1.07 \mathrm{~m} /$ day to 2.14 $\mathrm{m} /$ day, the condensate phase did not exhibit any rate sensitivity at high IFT's; however, as the IFT decreased to $0.01 \mathrm{~m}$ $\mathrm{N} / \mathrm{m}$, condensate relative permeability increased slightly. Similarly, for the experiments with irreducible water saturation, gas phase relative permeability sensitivity was more pronounced compared to condensate relative permeability sensitivity. The condensate relative permeability change; however, was more than ones where irreducible water saturation was zero. This can be explained with the fact that capillary pressures in the presence of connate water are systematically higher than the ones without water. The presence of a second liquid phase induces an "over capillarity" that depends on the IFT value and increases with gas saturation. This effect is practically zero for gas saturations below $20 \%$ $\mathrm{PV}$ and it is at a maximum in the vicinity of residual oil saturation [30]. The increase of "over capillarity" as IFT decreases may be explained by spreading coefficient, $\mathrm{C}$, which is defined as IFT (gas/water) - IIW (water/oil) - IFT (gas/oil) for a strongly water-wet system. If $\mathrm{C}$ is positive oil will spread between the other phases. On the other hand oil will not spread if $C$ is negative. Since $C$ is negative for the experiments with the presence of connate water saturation this may explain the greater effect of connate water on relative permeabilities.

These results can be interpreted using the condensate number concept even though it varies during the experiment. In a constant injection rate experiment, the condensate number first increases and then decreases as the resistance to flow reaches a maximum at intermediate saturations. Nevertheless, to distinguish between different experiments an average condensate number is assigned as shown in Table $\mathbf{2}$. It 

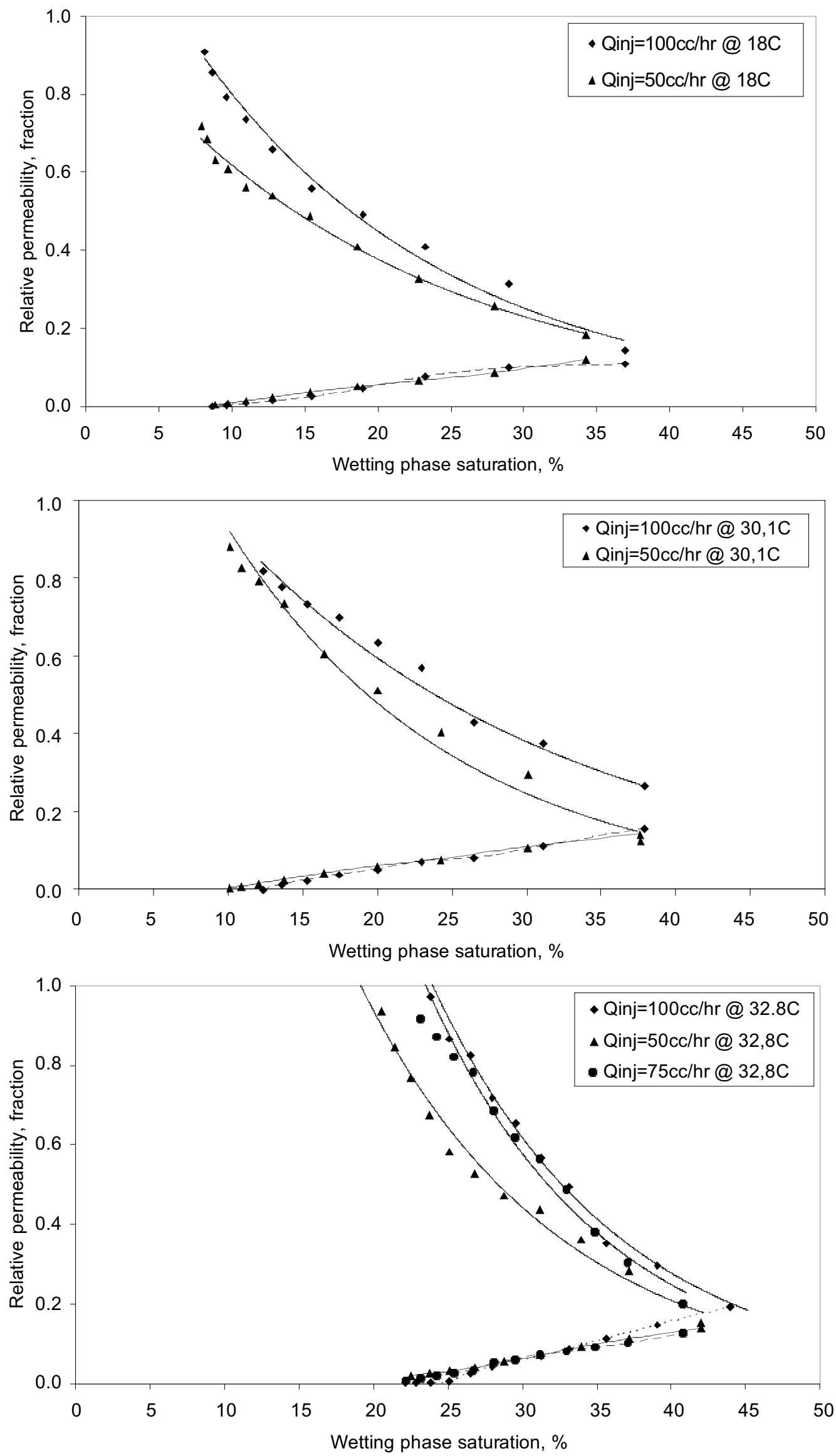

Fig. (6). Effect of flow rate at different IFT (Top: 0.3933, Middle: 0.059, Bottom: $0.01 \mathrm{~m} \mathrm{~N} / \mathrm{m}$ ); no water saturation. 

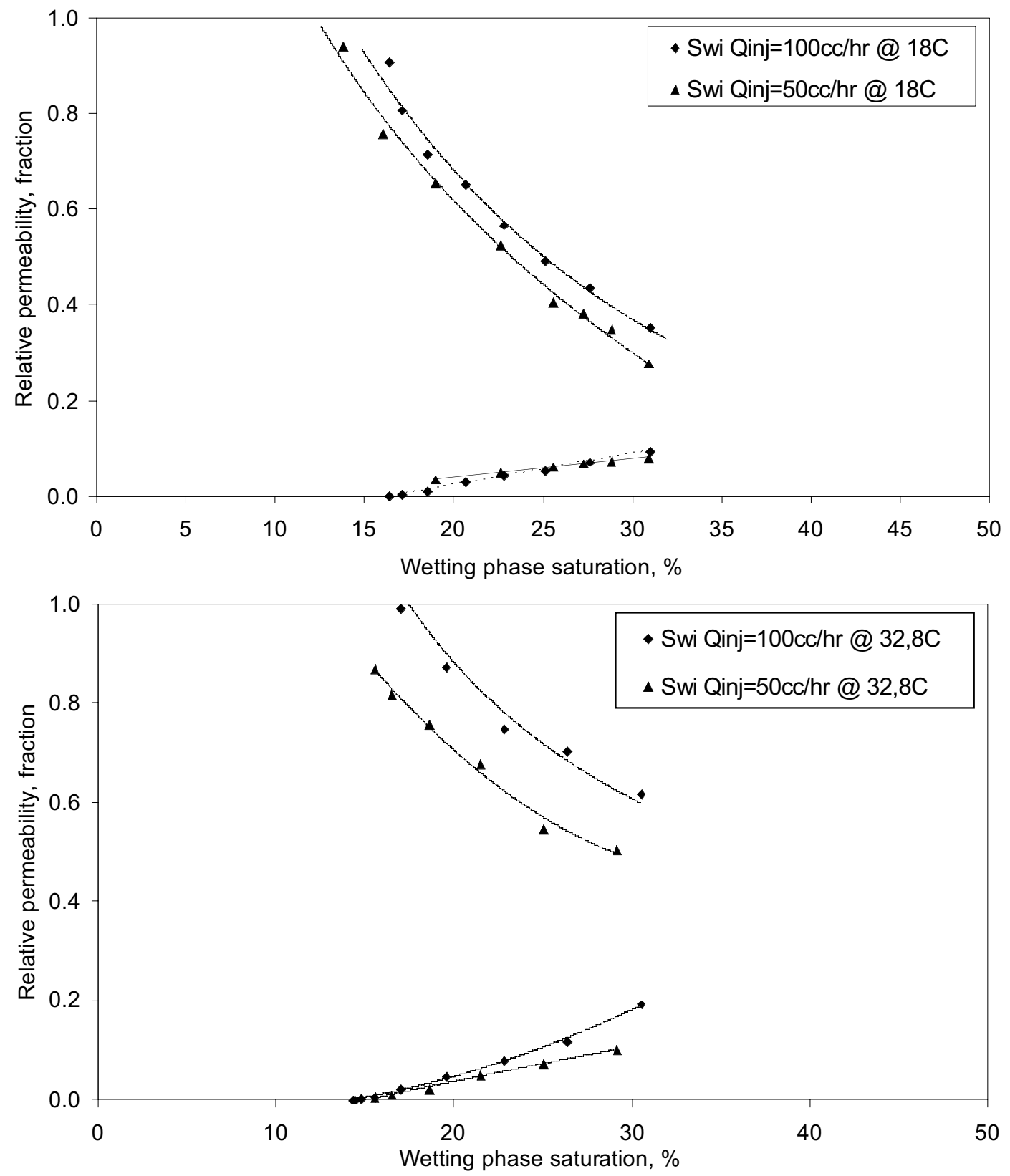

Fig. (7). Effect of flow rate at different IFT (Top: 0.3933, Middle: 0.059, Bottom: $0.01 \mathrm{~m} \mathrm{~N} / \mathrm{m}$ ); irreducible water saturation.

can be seen from Figs. (6-9) that as the condensate number increases the relative permeability of the gas phase increases. For drainage gas/condensate relative permeabilities Henderson et al. [27] reported that the relative permeability of the gas phase was particularly sensitive to velocity, and increased with increasing velocity at both values of IFT. Moreover the condensate phase relative permeabilities exhibited a degree of rate sensitivity for three tests at an IFT value of $0.14 \mathrm{~m} \mathrm{~N} / \mathrm{m}$, with the velocity ranging from 18.5 to $74 \mathrm{~m} /$ day. Similarly, Blom and Hagoort [14] reported that for low IFT experiments conducted using glass beads, the effect of increasing the superficial velocity by a factor of 2.5 was considerable. When the residual oil saturations were examined it was found that for cases where water was not introduced into the core plug as $\mathrm{N}_{\mathrm{k}}$ decreased residual wetting phase saturation decreased. It was observed that presence of an irreducible saturation did not change the results except at one point which may be due to an experimental error (Table 2). These results suggest that gas condensate flow in carbonates resemble the condensate flow in sandstones and glass bead packs.

\section{Effect of Interfacial Tension}

The experiments performed at higher temperature (i.e. $32.8^{\circ} \mathrm{C}$ ) give relative permeability curves to fluids with a lower IFT. The effect of varying IFT is summarized in Fig. (8) (zero irreducible water saturation) and Fig. (9) (irreducible water saturation). These figures show a clear dependence of relative permeability on interfacial tension regardless of presence of irreducible water saturation. The relative permeability to the non-wetting case increases gradually when the interfacial tension decreases by a factor of 39.33. At very low IFT the non-wetting relative permeability approaches a linear line for which the non-wetting relative permeability is 

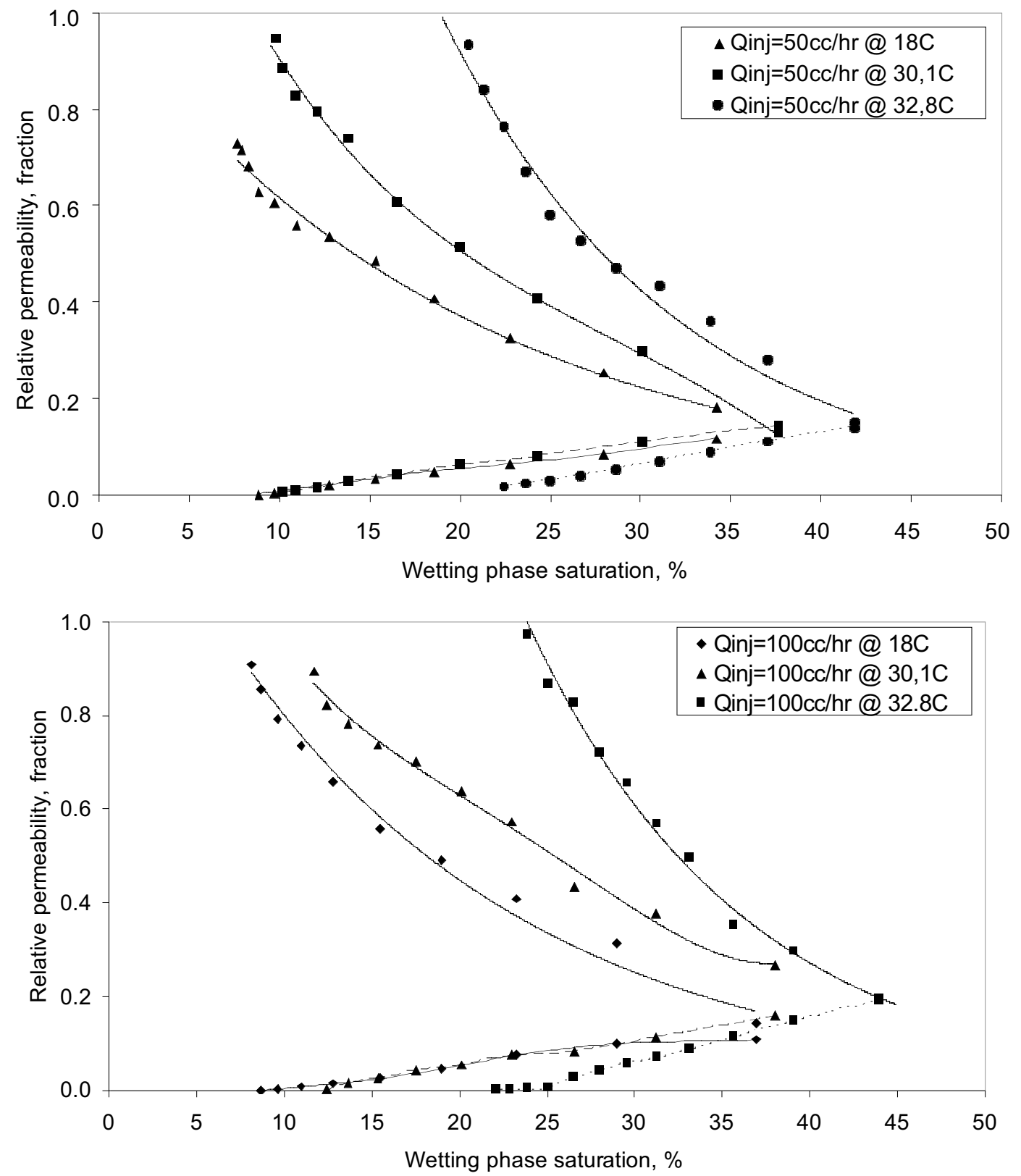

Fig. (8). Effect of IFT at different rates (Top: $50 \mathrm{cc} / \mathrm{hr}$, Bottom: $100 \mathrm{cc} / \mathrm{hr}$ ); no water saturation.

simply equal to the non wetting phase saturation. On the other hand, the wetting phase relative permeability is affected only at IFT values below $0.059 \mathrm{~m} \mathrm{~N} / \mathrm{m}$. The measured wetting phase relative permeability is quite low, so it is difficult to mark differences between the experiments. This can be due to the fact that the condensate number was not high enough. These results point to a threshold interfacial tension as was reported previously by $[1,3,11,12]$. The irreducible wetting phase saturation changes at IFT values below 0.059 $\mathrm{m} \mathrm{N} / \mathrm{m}$ when there is no immobile water saturation. However, when there is an immobile water phase the wetting phase irreducible saturation does not change significantly.

\section{MODELING}

Using the developed relative permeability model, the experiments are modeled. The model parameters $\mathrm{a}, \mathrm{b}$, and $\mathrm{c}$ were obtained by minimizing the least squares difference between the experimental and model relative permeability values the using Microsoft Excel's non-linear minimization algorithm. Figs. (6-9) show several examples of the matches obtained for different condensate numbers. It was observed that condensate relative permeability matches were much better compared to the gas ones. The mean square error was in the order of about $10^{-5}$ for condensate phase; however, it was calculated as $10^{-1}$ or $10^{-2}$ for gas phase. This shows that condensate number that incorporates both the capillary and bond numbers can be used to model condensate relative permeability. On the other hand, for gas relative permeability a different functional form may be needed. Although, not studied, logarithmic representation could be possibly used. These results suggest that regardless of the rock type a mathematical model can be used to express gas/condensate relative permeabilities as a function of condensate number. 

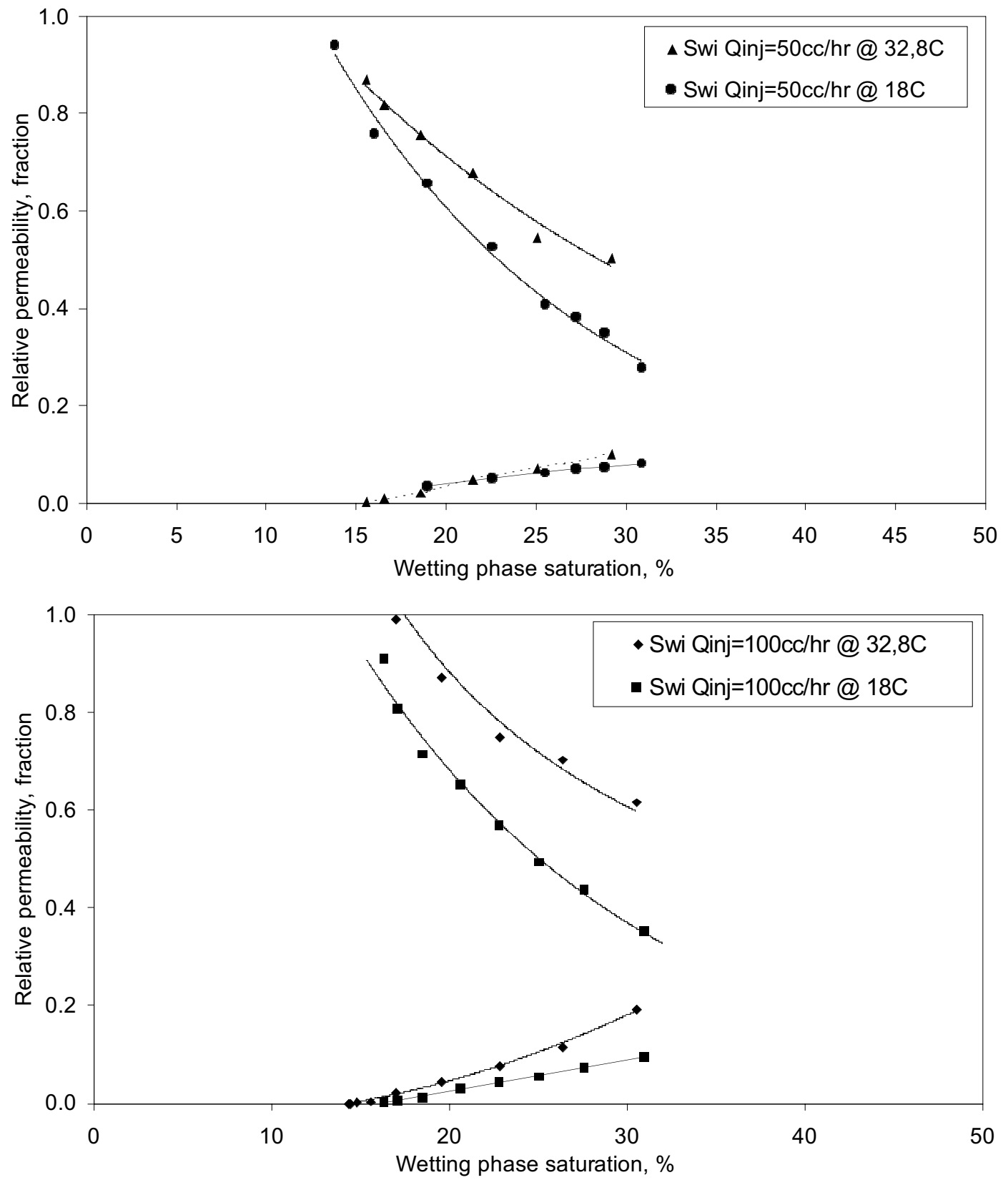

Fig. (9). Effect of IFT at different rates (Top: $50 \mathrm{cc} / \mathrm{hr}$, Bottom: $100 \mathrm{cc} / \mathrm{hr}$ ); irreducible water saturation.

\section{DISCUSSION}

Relative permeability accounts for the loss of effective flow area due to the fact that part of the saturation no longer contributes to the conductivity when a second or third phase is present. Moreover, it accounts for the increased tortuosity of the flow paths due to the presence of the additional phase(s). In this regard, both a decrease in IFT and an increase in flow velocity result in an increased permeability suggest that gas/condensate relative permeability data can be interpreted in terms of a balance between viscous, capillary and gravity forces. An increase of viscous forces compared to capillary forces a change in phase distribution in the pores such that the tortuosity of the flow path decreases which in turn reduces non-conductive saturation. Moreover, under capillary-dominated flow conditions, if the magnitude of the viscous forces is high enough short cut locations where one of the phases impedes a shorter way for the other mobile phases may form. Thus, mobilized yet isolated blobs that may contribute to the residual saturation may form. It has been previously observed that when one fluid strongly wets the porous medium, it forms a continuous network covering the entire solid surface [31]. Consequently, when the pressure drops below the dew point pressure and a small amount condensate is formed in such an environment, it may behave in one of three ways [32]:

- $\quad$ remain as a lens, i.e., a thick layer of finite extent,

- $\quad$ spread as a thin film until it is uniformly distributed over the water surface, or

- $\quad$ spread as a monolayer, the remaining excess collecting in a lens. 
The condensate spreads over the water surface completely when the spreading coefficient is equal to or greater than zero. If the condensate does not spread uniformly over the water surface which is probably the case for carbonates, it may form lenses and thus affecting the whole system. Such lenses may lead to large values of critical condensate saturation in the reservoir. Coskuner [33] reported isolated lenses in high pressure glass micro model experiments conducted using methane/n-pentane mixture. These results indicate that the capillary number alone can not be used to describe this complex behavior justifying the use of both capillary and bond numbers in the form of condensate number.

In addition, for simplicity and clarity, non-Darcy effects are not included in this illustrative study for carbonates. For very high rate gas wells, non-Darcy flow can be significant and is also coupled with all of the aforementioned variables and should be taken into account.

\section{CONCLUSIONS}

A new experimental procedure that allows the measurement of relative permeability to near-miscible fluids in the presence of immobile water saturation is developed and tested using a carbonate core plug.

1. Gas/condensate relative permeability experiments conducted using a carbonate sample showed marked resemblances to experiments conducted in sandstones and glass bead packs.

2. The results show a strong dependence of gas relative permeability on interfacial tension and superficial velocity. In the presence of immobile water saturation this dependence is more pronounced.

3. Capillary number alone can not be used to describe complex behavior of the gas/condensate flow.

4. When the experiments are interpreted in terms of a condensate number that is a sum of capillary and bond numbers, it was seen that the condensatenumber dependence of relative permeability differs for the two phases. The relative permeability to the non-wetting phase is affected at lower values of the condensate number.

\section{NOMENCLATURE}

\section{Latin}

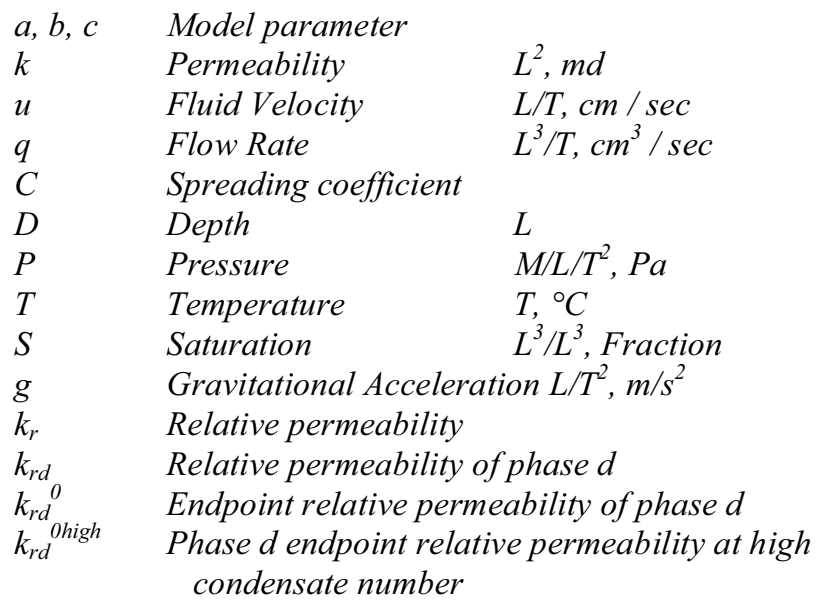

\begin{tabular}{|c|c|}
\hline$k_{\text {rd }}^{\text {olow }}$ & $\begin{array}{l}\text { Phase d endpoint relative permeability at low } \\
\text { condensate number }\end{array}$ \\
\hline$S_{d r}$ & Residual Saturation of phased $\quad, L^{3} / L^{3}, P V$ \\
\hline$S_{d r}^{h i g h}$ & Residual saturation of phase $d$ at high $N_{K}$ \\
\hline$S_{d r}^{\text {low }}$ & Residual saturation of phase $d$ at low $N_{K}$ \\
\hline$N_{c}$ & Capillary number \\
\hline$N_{b}$ & Bond number \\
\hline$N_{k}$ & Condensate number \\
\hline$x$ & Mole fraction \\
\hline$n$ & Number of the phases \\
\hline
\end{tabular}

Greek

$\begin{array}{lll}\sigma & \text { Interfacial Tension } & M T^{2}, \mathrm{mN} / \mathrm{m} \\ \mu & \text { Dynamic Viscosity } & M /(L T), \text { Pa.s } \\ \phi & \text { Porosity } & L^{3} / L^{3}, P V \\ \sigma_{d u} & \text { Interfacial tension of phases d and u } M / T^{2}, \mathrm{mN} / \mathrm{m} \\ \nabla \Phi_{d} & \text { Flow potential gradient } \\ \rho_{d} & \text { Density of phased } & M / L^{3}, \mathrm{~g} / \mathrm{cm} 3 \\ \Phi_{d} & \text { Potential of phase d } & M / L / T^{2}, P a\end{array}$

\section{Subscripts}

$\begin{array}{ll}n w & \text { non-wetting phase } \\ w & \text { wetting phase } \\ u & \text { Displaced phase } \\ d & \text { Displacing phase }\end{array}$

\section{REFERENCES}

[1] J. O. Ameafule, L. L. Handy, "The Effect of Interfacial Tensions on Relative Oil/Water Permeabilities of Consolidated Porous Media," SPEJ, vol. 371; Trans., AIME, 273, June 1982.

[2] L. W. Harbert, "Low Interfacial Tension Relative Permeability," Paper SPE 12171, SPE Annual Technical Conference and Exhibition, San Francisco, 5-8 October, 1983.

[3] G. D. Henderson, A. Danesh, D. H. Tehrani, S. Al-Shaidi, J. M. Peden, "Measurement and Correlation of Gas Condensate Relative Permeability by the Steady-State Method," SPEJ, vol 1, no. 2, pp. 191-201, January 1996.

[4] H. Asar, and L. L. Handy, "Influence of Interfacial Tension on Gas/ Oil Relative Permeability in a Gas-Condensate System," SPERE, 57, February 1998.

[5] D. S. Schechter, J. M. Haynes, "Relative Permeabilities of a NearCritical Binary Fluid". Transp. Porous Media, vol 9, pp. 241, 1992.

[6] J. Delclaud, J. Rochon, A. Nectoux, "Investigation of Gas/Oil Relative Permeabilities: High-Permeability Oil Reservoir Application," Paper SPE 16966, SPE Annual Technical Conference and Exhibition, Dallas, 27-30 September, 1987.

[7] F. J. M. Kalaydjian, B. J. Bourbiaux, J. M. Lombard, "Predicting Gas Condensate Reservoir Performance: How Flow Parameters Are Altered When Approaching Production Wells," Paper SPE 36715, SPE Annual Technical Conference and Exhibition, Denver, Colorado, 6-9 October, 1996.

[8] R. A. Fulcher, T. Ertekin, C. D. Stahl, "Effect of Capillary Number and Its Constituents on Two-Phase Relative Permeability Curves," $J P T$ vol. 249; Trans. AIME, 279, February 1985.

[9] D. S. Schechter, "Immiscible Flow Behavior in Porous Media". $\mathrm{PhD}$ dissertation, Universsity of Bristol, United Kingdom, 1988.

[10] W. Boom, K. Wit, A. M. Schulte, S. Oedai, J. P. W. Zeelenberg, and J. G. Maas, "Experimental Evidence for Improved Condensate Mobility at Near-Wellbore Flow Conditions," Paper SPE 30766, 1995 SPE Annual Technical Conference and Exhibition, Dallas, 667-675, 22-25 October, 1995

[11] C. Bardon, and D. G. Longeron, "Influence of Very Low Interfacial Tensions on Relative Permeability," SPEJ, vol. 391; Trans. AIME, 269, October 1980.

[12] G. R. Jerauld, "General Three-Phase Relative Permeability Model for Prudhoe Bay," SPERE, 255, November 1997.

[13] S. M. P. Blom, J. Hagoort, "How To Include the Capillary Number in Gas Condensate Relative Permeability Functions?," Paper SPE 
49268, SPE Annual Technical Conference and Exhibition, New Orleans, 27-30 October 1998a.

[14] Blom, S. M. P., Hagoort, J., "The Combined Effect of Near-Critical Relative Permeability and Non-Darcy Flow on Well Impairment by Condensate Drop-Out", SPERE, vol. 1, no. 5, pp. 421, October, $1998 b$.

[15] M. S. Haniff, J. K. Ali, "Relative Permeability and Low Tension Fluid Flow in Gas Condensate Systems," Paper SPE 20917 SPE Europec, The Hague, The Netherlands, 22-24 October, 1990.

[16] D. C. Morel, J.-F., Y. M. Morineu, A. G. Putz, "Mobility of Hydrocarbon Liquids in Gas Condensate Reservoirs: Interpretation of Depletion Laboratory Experiments," Paper SPE 24939, SPE Annual Technical Conference and Exhibition, Washington, DC, 4-7 October, $875-886,1992$.

[17] K. J. Hartman, and A. S. Cullick, "Oil Recovery by Gas Displacement at Low Interfacial Tension," J. Petrol. Sci Eng.,vol. 10, pp. 197,1994

[18] P. K. Munkerud, "The Effects of Interfacial Tension and Spreading on Relative Permeability in Gas Condensate Systems," Proc., 8th European IOR Symposium, 1995.

[19] J. W. Cahn, "Critical Point Wetting," J. Chem. Phys. vol. 66, no. 8, pp. $3667,1977$.

[20] J. K. Williams, A. R. Dawe, "Near-Critical Condensate Fluid Behavior in Porous Media - A Modeling Approach," SPE 17137, SPE Reservoir Eng., 221-228, May 1989.

[21] O. R. Wagner, R. O. Leach, "Effect of Interfacial Tension on Oil Displacement Efficiency," SPEJ, 335 Trans., AlME, 237, December 1966.

[22] K. H. Coats, "An Equation of State Compositional Model," SPEJ, vol. 20 , pp. $363,1980$.

[23] L. E. Brownell and D. L. Katz, "Flow of Fluids through Porous Media-Part II,” Chem. Eng. Prog., vol. 43, no. 11, pp. 601, 1947.

[24] M. Delshad, D. Bhuyan, G. A. Pope, L. W. Lake, "Effects of Capillary Number on the Residual Saturation of Three Phase Micellar
Solution," Paper SPE 14911, SPE/DOE Symposium on Enhanced Oil Recovery, Tulsa, Oklahoma, 20-23 April, 1986.

[25] G. A. Pope, W. Wu, G. Narayanaswamy, M. Delshad, M. Sharma, P. Wang, "Modeling Relative Permeability Effects in Gas Condensate Reservoirs," Paper SPE 49266, SPE Annual Technical Conference and Exhibition, New Orleans, 27-30 September, 1998.

[26] M. Jin, "A Study of Non-Aqueous Phase Liquid Characterization and Surfactant Remediation," PhD dissertation, The University of Texas, Austin, Texas, 1995.

[27] G. D. Henderson, A. Danesh, B. Al-kharusi, D. Tehrani, "Generating reliable gas condensate relative permeability data used to develop a correlation with capillary number," J. Petrol. Sci. Eng., vol. 25, 79-91, 2000 .

[28] H. Calisgan, "Comprehensive Modelling of Gas Condensate Relative Permeability and its Influence on Field Performance," PhD dissertation, Middle East Technical University, Ankara, Turkey, 2005

[29] S. Akin, and B. Demiral, "Genetic Algorithm for Estimating Relative Permeabilities from Displacement Experiments," Comput. Geosci., vol. 24, no. 3, pp. 251-258, 1998.

[30] D. G. Longeron, F. Kalaydjian, C. Bardon, L. M. Desremaux, "Gas/Oil Capillary Pressure: Measurements at Reservoir Conditions and Effect on Gas-Gravity Drainage," Paper SPE 28612, 69th ATCE, New Orleans, 1994.

[31] F. A. L. Dullien, C. Zarcone, I. F. MacDonald, A. Collin, R. D. F. Bochard, "The effects of surface roughness on the capillary pressure curves and the heights of capillary rise in glass bead packs," $J$. Coll Int. Sci.,vol. 127 no. 2, 362-372, 1989.

[32] A. W. Adamson, Physical Chemistry of Surfaces, John Wiley, New York, 1976.

[33] G. Coskuner,"Microvisual Study of Multiphase Gas Condensate Flow in Porous Media," Trans. Porous Media, vol. 28, 1-18, 1997.

(C) Calisgan and Akin; Licensee Bentham Open.

This is an open access article distributed under the terms of the Creative Commons Attribution License (http://creativecommons.org/licenses/by/2.5/), which permits unrestrictive use, distribution, and reproduction in any medium, provided the original work is properly cited. 